\title{
Comparison of conventional versus single port laparoscopy for surgical treatment of gynecological diseases: a pilot study
}

\author{
Ying Zhang, Yingjun Zhu \\ Department of Gynecology, Tianjin Central Hospital of Gynecology Obstetrics, Tianjin, China
}

Videosurgery Miniinv 2022; 17 (1): 252-260

DOI: https://doi.org/10.5114/wiitm.2021.105823

\begin{abstract}
Introduction: Many recent studies have conducted laparoscopic single-site surgery (LESS) using single-port laparoscopy (SPL), which combines conventional laparoscopy (CL) with a novel multichannel port. However, to implement SPL, several obstacles must be overcome.

Aim: To study the clinical value of SPL in the surgical treatment of gynecological diseases.

Material and methods: Twenty-five patients with ectopic pregnancy (EP) and 11 with uterine leiomyoma (UL) were randomly assigned to undergo either LESS by SPL or CL. The CL was performed routinely, while the SPL was performed through a single port using a self-made, multi-channel laparoscopic approach based on CL. The following parameters were compared between the SPL and CL groups: intraoperative conditions (operation time and blood loss), postoperative conditions (exhaustion and hospital stay time), and visual analog scale. Patients with EP and those with UL were analyzed separately in this regard. In patients with UL, hemoglobin changes, complications, and long-term physical recovery within 6 months of surgery were also compared.

Results: The operation time was significantly longer in the SPL group than in the CL group $(p<0.001)$. However, blood loss, postoperative exhaustion, and hospital stay time were significantly lower ( $p<0.05$ in all cases). In patients with UL, intraoperative and postoperative conditions did not differ significantly between the groups. At the follow-up within 6 months, patients with UL in the SPL group had recovered, with better cosmetic effects and more satisfaction. No cases of umbilical incisional hernia occurred in the SPL group.

Conclusions: SPL showed clinical efficacy, with minimal invasion, rapid recovery, and cost-effectiveness in patients with EP or UL.
\end{abstract}

Key words: single-port laparoscopy, conventional laparoscopy, ectopic pregnancy, uterine leiomyoma.

\section{Introduction}

Ectopic pregnancy (EP) accounts for approximately $2 \%$ of all pregnancies [1], and $95 \%$ of EPs occur in the Fallopian tube. The ampullary part of the tube is the most common site, but the remainder of the tube, including the abdomen, ovary, and cervix/ hysterotomy scar, have also been reported as sites of EP [2, 3]. Patients with EP are preliminarily diagnosed using series titers of human chorionic gonad- otrophin ( $\mathrm{hCG}$ ) evaluated every 2 days; they are then definitively diagnosed using ultrasound. When the site of implantation is still unclear on sonography, laparoscopy can be used to ascertain the site and perform the operation at the same time [4]. Laparoscopy has replaced laparotomy as the first-line therapy for EP; it has better safety and efficacy, involving less blood loss and discomfort, fewer complications after complete resection, shorter hospital stays, and a recovery time of $X Y Z$ [5]. 
The other disease studied in the present project was uterine leiomyoma (UL) - a benign tumor of the uterine smooth muscle cells whose incidence varies from $5.4 \%$ to $77.0 \%$. It is difficult to state the exact incidence of UL because patients often conceal symptoms and may even be symptom-free [6]. ULs in the uterus can cause infertility or adverse pregnancy outcomes $[7,8]$. The Nurses' Health Study showed that hysterectomy or bilateral oophorectomy can elevate the mortality of $U L$ in patients under 50 years who have never received estrogen therapy [9]. Although most patients still insist on conservative treatments with no surgery, such approaches often fail. Thus, uterus-conserving treatments using abdominal, laparoscopic, or hysteroscopic myomectomy are recommended [10]. Such minimally invasive surgery is cost-effective and involves short fertility recovery times [11].

Laparoscopes have developed rapidly and now combine many novel techniques. They often possess flexible tips or three-dimensional imaging systems, which provide a broad visual and operation area. Unlike conventional laparoscopy (CL), single-port laparoscopy (SPL) involves inserting multiport trocars through a single incision of the umbilicus [12]. This innovation adds a multichannel, single-port system and articulating instruments to the $\mathrm{CL}$. After suturing of the umbilicus and neonatal scar, the operation leaves virtually no scars [13]. However, to implement SPL, several obstacles must be overcome, including reduced visualization, loss of triangulation, and instrument interference [14]. To overcome the problem of trocar interference, a flexible articulated instrument that is airtight at the incision of the umbilicus must be used. Fixation stability of this instrument on the abdomen is also important to ensure that relatively large specimens can be extracted from the umbilicus using rotatory resection [15]. That said, it has been reported that this single-use, expensive instrument can be replaced by a surgical glove to relieve collisions without additional costs in laparoscopic single-site surgery (LESS) [16].

\section{Aim}

In the present study, because articulated instruments are expensive, we used a surgical glove with reasonable changes, which allowed patients with both EP and UL to undergo LESS via SPL. To inves- tigate whether this self-made SPL could be applied in clinical practice, we compared intraoperative and postoperative outcomes, as well as long-term physical recovery, between LESS and $\mathrm{CL}$ in these patients. This study was approved by the Ethics Committee of Tianjin Central Hospital of Gynecology Obstetrics.

\section{Material and methods}

\section{Patients}

A total of 36 patients were included, including 25 with EP and 11 with UL who were treated in our organization between June 2019 and December 2019. Based on medical history, physical signs, blood hCG, and pelvic ultrasound, 25 patients were highly suspected to have EP and signed an informed consent form before the operation. After excluding those with contraindications to laparoscopic surgery, we randomly assigned the patients with EP to either the SPL group $(n=12)$ or the $\mathrm{CL}$ group $(n=$ 13). Eleven patients with UL, who were treated in the same department, were also enrolled and signed informed consent forms before the operation. The inclusion criteria for patients with UL were as follows: (1) subserous, intermural, or broad ligament UL with a diameter $\leq 10 \mathrm{~cm}$ and a leiomyoma number $\leq 5$; (2) no degeneration of leiomyoma on ultrasound and no abnormality of tumor biomarkers in pathology; (3) no surgical contraindications. The exclusion criteria were as follows: (1) body mass index (BMI) $\geq 30 \mathrm{~kg} / \mathrm{m}^{2}$; (2) infected wound in the umbilicus; (3) scar constitution; (4) extensive pelvic adhesion found during intraoperative exploration. Patients with UL were randomly assigned to the SPL $(n=5)$ or $\mathrm{CL}(n=6)$ groups.

\section{Laparoscopic surgery}

Patients with EP were treated by lesion resection of the EP followed by ovarian cyst elimination. A trocar with a $10-\mathrm{mm}$ diameter and $0^{\circ}$ angle (Olympus Corp., Japan) was inserted into the first incision in the umbilicus (approximately $10 \mathrm{~mm}$ ). A trocar with a $5-\mathrm{mm}$ diameter was then inserted into the second incision (approximately $5 \mathrm{~mm}$ ) at 2-3 fingers above the anterior superior iliac spine of the left lower abdomen. Another trocar with a 5-mm diameter was inserted into the third incision (approximately $5 \mathrm{~mm}$ ), which was made between the first and sec- 
ond incisions. Under these laparoscopies, the Fallopian tube was coagulated using bipolar forceps and completely cut along the mesosalpinx to the side of the uterus using scissors. It was packed into a specimen bag, which was manually removed through the umbilical incision using rotatory resection. After the surgical incision was examined and the bleeding was stopped, the pelvis was washed and protected using special anti-adhesion materials. The skin of the umbilical incision was closed using buried sutures with 603 absorbable lines.

Routine myomectomy was also performed using $\mathrm{CL}$ in patients with UL. In addition to the three trocars and respective incisions that were used in the patients with EP, a fourth incision (approximately $5 \mathrm{~mm}$ ) was made at McBurney's point and a 5-mm diameter trocar was inserted. After injection of vasopressin at the junction of the tumor and uterus to promote contraction of the uterus, the UL was removed using a unipolar electric hook at the vertical and inclined incision on the most prominent surface. The tumor cavity and uterine incision were then continuously sutured and closed using a fishbone line

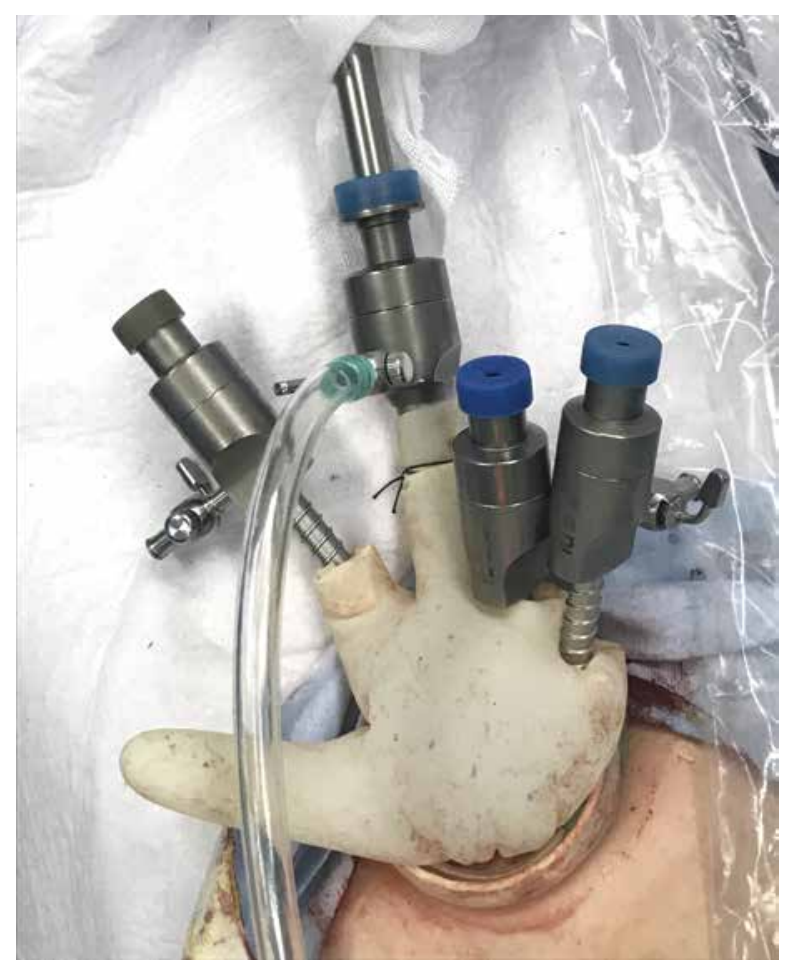

Photo 1. The self-made, single-port laparoscopic devices were positioned as shown. The ports were inserted through a single incision at the umbilicus
(Johnson Company), the second layer of which was sutured. The UL was packed into a specimen bag, which was manually removed through the expanded third incision (approximately $10 \mathrm{~mm}$ ) using rotatory resection. After the surgical incision was examined and the bleeding was stopped, the pelvis was washed and protected using special anti-adhesion materials. The continuous layers of the expanded third incision were sutured using absorbable lines, and the skin of both the third incision and the umbilical incision was closed using buried sutures with 603 absorbable lines.

\section{Single-port laparoscopy using a self-made device}

Two sections of disposable suction tubes $8-10 \mathrm{~cm}$ long were cut as the supporting ring; both were self-connected from head to tail to form rings, one of which was directly flipped over and fixed at the cuff of a surgical glove, while the other was fixed at the thumb of the glove and flipped through the first ring. The distance between the two rings could be adjusted to accommodate the thickness of the patient's skin. A trocar with a $10-\mathrm{mm}$ diameter was inserted into the middle finger of the glove, while a trocar with a 5-mm diameter was inserted into the index, ring, or little fingers of the glove.

\section{LESS technique}

LESS was conducted using a self-made, multichannel laparoscopic device through a single incision (Photos 1 and 2). Patients were placed in the bladder lithotomy position and had a urinary catheter. The self-made, single-port, multi-channel laparoscopic devices were inserted through an incision of $2.5-3 \mathrm{~cm}$ above the upper margin of the umbilicus. They were then fixed depending on abdominal pressure. The SPL was carried out using laparoscopy with a 10-mm diameter and $0^{\circ}$ angle (Olympus Corp., Japan), CL instruments (Kangji Medical Equipment Co., Ltd.), and bipolar coagulation (Kangji Medical Equipment Co., Ltd.). The procedure for removing the Fallopian tube or UL was identical to that of the laparoscopic surgery. The Fallopian tube or UL was packed into a specimen bag, which was manually removed through the umbilical incision using rotatory resection. After the surgical incision was examined and the bleeding was stopped, the pelvis was washed and protected using special anti-adhesion materials. The continuous layers of the umbilical incision were sutured using 2-0 absorbable lines and closed; the 
skin of the umbilical incision was cosmetically sutured using 4-0 skin needles. The SPL and CL procedures were performed by the same physician.

\section{Evaluation indicators}

The intraoperative conditions (operation time and blood loss), postoperative conditions (exhaustion and hospital stay time), and visual analogue scale (VAS) score were recorded in both groups. In patients with UL, both intraoperative and postoperative hemoglobin levels, complications, and long-term physical condition at 1, 3, and 6 months after surgery were collected.

The VAS score ranged from 0 to 10 , which indicated no pain at 0 points, tolerable mild pain at $0-$ 3 points, pain affecting sleep at 4-6 points, and severe pain affecting appetite at $7-10$ points [17].

\section{Ethics approval and consent to participate}

This study was approved by the Ethics Committee of Tianjin Central Obstetrics and Gynecology Hospital and informed consent was obtained from each patient or candidate.

\section{Statistical analysis}

Statistical analysis was performed using SPSS 18.0. Continuous variables were expressed as mean \pm standard deviation and analyzed using the $t$-test. Mean difference (MD) with $95 \%$ confidence interval (CI) was used to evaluate differences between the groups. Categorical variables were analyzed using the $\chi^{2}$ test. Odds ratio (OR) with $95 \% \mathrm{Cl}$ was used to evaluate the difference between the groups. Statistical significance was set at a $p$-value of $<0.05$.

\section{Results}

\section{Baselines of patients with EP or UL}

The average age of the patients with EP in the SPL group was $34.5 \pm 6.5$ years (range: $19-41$ years), while it was $36.7 \pm 7.2$ years (range: $20-40$ years) in the $\mathrm{CL}$ group. The mean BMI was $22.6 \pm 3.6 \mathrm{~kg} / \mathrm{m}^{2}$ in the SPL group and $21.7 \pm 4.1 \mathrm{~kg} / \mathrm{m}^{2}$ in the $\mathrm{CL}$ group. With regards to the site of the EP, the SPL group included 12 cases of tubal pregnancy and no cases of ovarian pregnancy. Three of the patients had concomitant ovarian cysts. The $\mathrm{CL}$ group included 12 tubal pregnancies, of which two were combined with ovarian cysts. There were no significant differ-

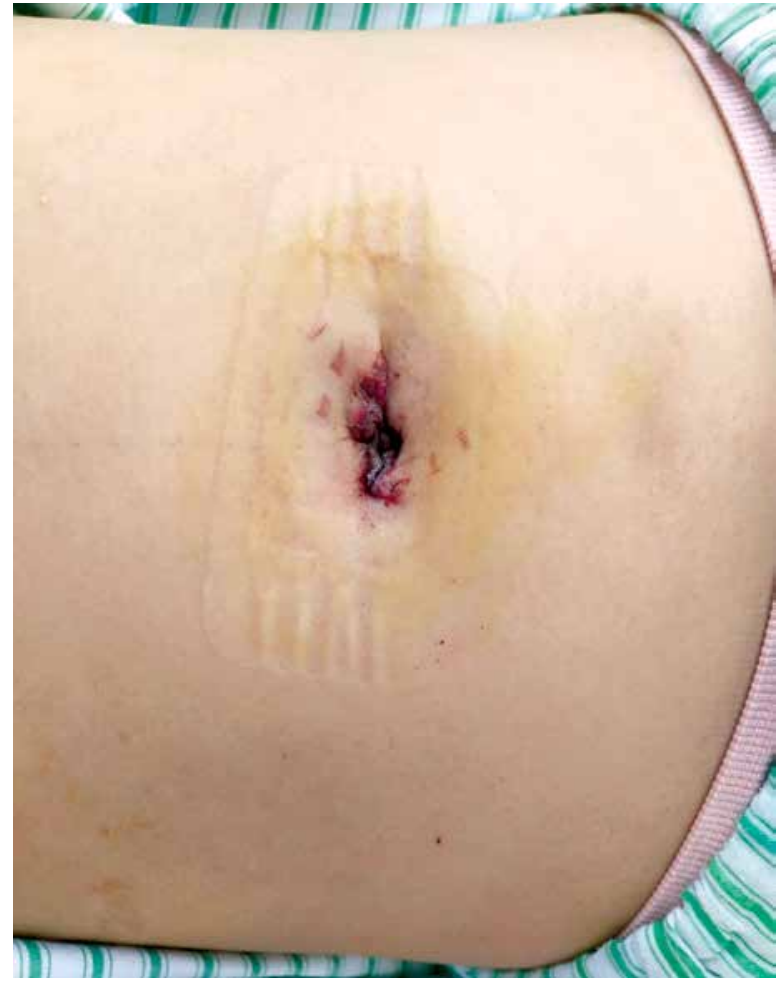

Photo 2. The skin incision of the umbilicus was cosmetically sutured using 4-0 skin needles

ences in the baseline values of patients with EP, including age, $\mathrm{BMI}$, and type of pregnancy $(p>0.05$ in all cases; Table I).

In patients with UL, the average age of the SPL group was $34.5 \pm 5.5$ years, while that of the $\mathrm{CL}$ group was $35.5 \pm 5.2$ years. The mean BMI was 23.6 $\pm 4.5 \mathrm{~kg} / \mathrm{m}^{2}$ in the $\mathrm{SPL}$ group and $22.6 \pm 4.8 \mathrm{~kg} / \mathrm{m}^{2}$ in $\mathrm{CL}$ group. Regarding the history of abdominal surgery, 1 patient in the SPL group had undergone cesarean section, one had undergone laparoscopic ovarian cystectomy, and one had undergone appendectomy. In the CL group, 3 patients had undergone cesarean section, two had undergone laparoscopic ovarian cystectomy, and one had undergone cholecystectomy. The main comorbidities were high blood pressure (2 patients each in the SPL and CL groups), diabetes (1 patient in the $\mathrm{CL}$ group), and hyperthyroidism (1 patient in the SPL group). The postoperative pathology reports of the patients were all diagnostic of UL. The mean maximum myoma diameter was $6 \pm 3.4 \mathrm{~cm}$ in the SPL group and $6 \pm 2.8 \mathrm{~cm}$ in the $\mathrm{CL}$ group. The average number of myomas was $2 \pm 1.6$ in the SPL group and $3 \pm 1.4$ in the $\mathrm{CL}$ group. The baseline demography and illness conditions 
Table I. Comparison of demographic and medical conditions of patients with ectopic pregnancy between single-port laparoscopy (SPL) group and conventional laparoscopy (CL) group

\begin{tabular}{|c|c|c|c|c|}
\hline Indicators & $\begin{array}{l}\text { CL group } \\
(n=13)\end{array}$ & $\begin{array}{l}\text { SPL group } \\
(n=12)\end{array}$ & MD or OR $(95 \% \mathrm{Cl})$ & $P$-value \\
\hline Age [years] mean \pm SD & $36.7 \pm 7.2$ & $34.5 \pm 6.5$ & $2.2(-3.5,7.9)^{5}$ & $0.483^{a}$ \\
\hline Body mass index $\left[\mathrm{kg} / \mathrm{m}^{2}\right]$ mean $\pm \mathrm{SD}$ & $21.7 \pm 4.1$ & $22.6 \pm 3.6$ & $-0.9(-4.1,2.3)^{5}$ & $0.659^{a}$ \\
\hline Positions of pregnancy, $n(\%)$ : & & & $0.33(0.01,8.99)^{\#}$ & $0.340^{b}$ \\
\hline Tubal & $12(92.3)$ & $12(100.0)$ & & \\
\hline Ovarian & $1(7.7)$ & $0(0.0)$ & & \\
\hline Combined with ovarian cysts, $n(\%)$ & $2(15.4)$ & $3(25.0)$ & $0.55(0.07,4.01)^{\#}$ & $0.571^{b}$ \\
\hline
\end{tabular}

${ }^{a} P$-values were statistically calculated by $t$-test. ${ }^{b}$-values were statistically calculated by $\chi^{2}$ test. $M D$ - mean difference, $O R$ - odds ratio. ${ }^{5} \mathrm{MD}(95 \% \mathrm{Cl})$; ${ }^{\#}$ OR $(95 \% \mathrm{Cl})$.

Table II. Comparison of demographic and medical conditions of patients with uterine leiomyoma between single-port laparoscopy (SPL) group and conventional laparoscopy $(\mathrm{CL})$ group

\begin{tabular}{|c|c|c|c|c|}
\hline Indicators & $\begin{array}{l}\text { CL group } \\
(n=6)\end{array}$ & $\begin{array}{l}\text { SPL group } \\
\quad(n=5)\end{array}$ & MD or OR $(95 \% \mathrm{Cl})$ & $P$-value \\
\hline Age [years], mean \pm SD & $35.5 \pm 5.2$ & $34.5 \pm 5.5$ & $1.0(-6.3,8.3)^{5}$ & $0.548^{a}$ \\
\hline Body mass index $\left[\mathrm{kg} / \mathrm{m}^{2}\right]$ mean $\pm \mathrm{SD}$ & $22.6 \pm 4.8$ & $23.6 \pm 4.5$ & $-1.0(-7.4,5.4)^{5}$ & $0.672^{\mathrm{a}}$ \\
\hline \multicolumn{5}{|l|}{ History of abdominal surgery, $n(\%)$ : } \\
\hline Cesarean section & $3(50.0)$ & $1(20.0)$ & $4.00(0.27,60.32)^{\#}$ & 0.545 \\
\hline Laparoscopic ovarian cystectomy & $2(33.3)$ & $1(20.0)$ & $2.00(0.13,31.98)^{\#}$ & 0.620 \\
\hline Cholecystectomy & $1(16.7)$ & $0(0.0)$ & $3.00(0.10,90.96)^{\#}$ & 0.255 \\
\hline Appendectomy & $0(0.0)$ & $1(20.0)$ & $0.23(0.01,7.05)^{\#}$ & 0.455 \\
\hline \multicolumn{5}{|l|}{ Comorbidity, $n$ (\%): } \\
\hline High blood pressure & $2(33.3)$ & $2(40.0)$ & $0.75(0.06,8.83)$ & 0.819 \\
\hline Diabetes & $1(16.7)$ & $0(0.0)$ & $3.00(0.10,90.96)^{\#}$ & 0.255 \\
\hline Hyperthyroidism & $0(0.0)$ & $1(20.0)$ & $0.23(0.01,7.05)^{\#}$ & 0.455 \\
\hline $\begin{array}{l}\text { Maximum diameter of myoma }[\mathrm{cm}] \\
\text { mean } \pm \text { SD }\end{array}$ & $6 \pm 2.8$ & $6 \pm 3.4$ & $0(-4.2,4.2)^{5}$ & $0.876^{a}$ \\
\hline Number of myomas, mean \pm SD & $3 \pm 1.4$ & $2 \pm 1.6$ & $1(-1.0,3.0)$ & $0.685^{a}$ \\
\hline
\end{tabular}

aP values were statistically calculated by $t$-test. ${ }^{b} P$-values were statistically calculated by $\chi^{2}$ test. $M D-$ mean difference, OR - odds ratio. ${ }^{5} M D(95 \%$ CI); ${ }^{\#}$ OR $(95 \% \mathrm{Cl})$.

were comparable between the two groups $(p>0.05$ in all cases; Table II).

The intraoperative and postoperative conditions of SPL were better than those of $\mathrm{CL}$ in patients with $\mathrm{EP}$

All operations in patients with EP were successful, without any conversion to laparotomy. In patients with EP receiving LESS or laparoscopic surgery, the operation time was significantly longer in the SPL group than in the $\mathrm{CL}$ group $(\mathrm{MD}=-31.50,95 \% \mathrm{Cl}$ : $42.69,-20.31, p<0.05)$. Moreover, the intraoperative blood loss (MD $=4.33,95 \% \mathrm{Cl}: 0.74,7.92, p<0.05)$, postoperative exhaust time $(\mathrm{MD}=0.52,95 \% \mathrm{Cl}: 0.08$, $0.96, p<0.05)$, and hospital stay time $(\mathrm{MD}=1.02$, $95 \% \mathrm{Cl}: 0.49,1.55, p<0.05)$, and the VAS (MD $=0.83$, $95 \% \mathrm{Cl}: 0.24,1.42, p<0.05)$ in the SPL group were significantly lower than in the $\mathrm{CL}$ group (Table III). 
Table III. Comparisons of clinical indexes between single-port laparoscopy (SPL) group and conventional laparoscopy $(\mathrm{CL})$ group in patients with ectopic pregnancy

\begin{tabular}{|lcccc|}
\hline Indicators $^{*}$ & $\begin{array}{c}\text { CL group } \\
(n=13)\end{array}$ & $\begin{array}{c}\text { SPL group } \\
(n=12)\end{array}$ & MD (95\% CI) & $P_{\text {-value }}$ \\
\hline Operation time [min] mean \pm SD & $63.08 \pm 11.10$ & $94.58 \pm 15.73$ & $-31.50(-42.69,-20.31)$ & $<0.001$ \\
\hline Intraoperative blood loss [ml] mean \pm SD & $13.08 \pm 5.22$ & $8.75 \pm 3.11$ & $4.33(0.74,7.92)$ & 0.020 \\
\hline Postoperative exhaust time [days] mean \pm SD & $1.77 \pm 0.60$ & $1.25 \pm 0.45$ & $0.52(0.08,0.96)$ & 0.023 \\
\hline Hospital stay time [days] mean \pm SD & $3.69 \pm 0.75$ & $2.67 \pm 0.49$ & $1.02(0.49,1.55)$ & 0.001 \\
\hline Visual analogue score, mean \pm SD & $3.00 \pm 0.82$ & $2.17 \pm 0.58$ & $0.83(0.24,1.42)$ & 0.008 \\
\hline
\end{tabular}

aP-values were statistically calculated by t-test. $M D$ - mean difference.

Intraoperative and postoperative conditions and long-term recovery after SPL were comparable with those after $\mathrm{CL}$ in the UL group

In patients with UL undergoing LESS or laparoscopic surgery, both SPL and CL were successfully completed without any conversion to laparotomy or traditional porous laparoscopy that involved puncturing more holes. The operation time of the SPL group was slightly longer than that of the $C L$ group, but the difference was not significant. Furthermore, the intraoperative blood loss, postoperative exhaustion, hospital stay time, VAS score, and hemoglobin change were comparable between the two groups ( $p>0.05$ in all cases). However, the SPL group had postoperative complications, while the $\mathrm{CL}$ group did not (Table IV). The only postoperative complication that occurred in the present study was venous thrombosis in the lower extremity, which was assessed as unrelated to the LESS and resolved after anticoagulant therapy.
All patients had recovered well at 1, 3, and 6 months after surgery. Moreover, in the SPL group, the umbilical wound healed with better cosmetic effects and greater satisfaction. There were no complications of umbilical incisional hernia during the entire follow-up period.

\section{Discussion}

Both EP and UL are common gynecological diseases for which laparoscopic surgery has become the first-line therapy because it is minimally invasive [18]. Many recent studies have conducted LESS using SPL, which combines $\mathrm{CL}$ with a novel multichannel port. SPL has proven safe and effective in the early stages of cervical cancer and endometrial carcinoma $[19,20]$. The single incision of SPL has cosmetic advantages that improve satisfaction in both doctors and patients. In the present study, in which we used SPL in a novel setting to treat other common gynecological diseases, we found that the

Table IV. Comparisons of perioperative related indicators between single-port laparoscopy (SPL) group and conventional laparoscopy $(\mathrm{CL})$ group in patients with uterine leiomyoma

\begin{tabular}{|lcccc|}
\hline Indicators & $\begin{array}{c}\text { CL group } \\
(n=6)\end{array}$ & $\begin{array}{c}\text { SPL group } \\
(n=5)\end{array}$ & MD or OR (95\% Cl) & $P_{\text {-value }}$ \\
\hline Operation time [min] mean \pm SD & $75 \pm 56.9$ & $101 \pm 55.7$ & $-26(-103.2,51.2)^{5}$ & 0.466 \\
\hline Blood loss [ml] mean \pm SD & $55 \pm 37.9$ & $56 \pm 35.8$ & $-1(-51.7,49.7)^{5}$ & 0.376 \\
\hline Postoperative exhaust time [h] mean \pm SD & $14.6 \pm 4.4$ & $13.5 \pm 3.4$ & $1.1(-4.4,6.6)^{5}$ & 0.286 \\
\hline Hemoglobin change [g/l] & $3.1 \pm 2.7$ & $4.4 \pm 2.8$ & $-1.3(-5.1,2.5)$ & 0.455 \\
\hline Intraoperative complications, $n$ (\%) & $0(0.0)$ & $0(0.0)$ & NA & 1 \\
\hline Hospital stay time [days] mean \pm SD & $3.4 \pm 2.8$ & $3.5 \pm 2.6$ & $-0.1(-3.8,3.6)^{5}$ & 0.597 \\
\hline Visual analogue score, mean \pm SD & $2.1 \pm 1.3$ & $2.0 \pm 1.2$ & $0.1(-1.6,1.8)^{5}$ & 0.397 \\
\hline Postoperative complications, $n(\%)$ & $0(0.0)$ & $1(20.0)$ & $0.23(0.01,7.05)^{\#}$ & 0.455 \\
\hline
\end{tabular}

aP-values were statistically calculated by $t$-test. $N A$ - not available, $M D$ - mean difference, OR-odds ratio. ${ }^{5} \mathrm{MD}(95 \% \mathrm{Cl})$; ${ }^{\#} \mathrm{OR}(95 \% \mathrm{Cl})$. 
procedure showed better efficacy and safety both during and after surgery, and that long-term physical recovery showed more satisfactory cosmetic effects in patients with EP or UL.

Some commercially available multi-channel port devices are the GelPort, SILS port, and common single surgical port [21, 22]. Among these devices, only the TriPort, which is produced by Johnson \& Johnson, is approved in China. It has a low incidence of pneumoperitoneum, and the various optional angles of the ports can widen the manipulation area, making the procedure easier for surgeons [23, 24]. In addition, single ports using wound retractors allow a broader range of diameters and port dimensions. However, the maximum depth of the inserted TriPort is approximately 10 $\mathrm{cm}$, necessitating laparoscopic instruments at an additional cost [25]. Thus, the self-made, multi-channel laparoscopic surgical glove has obvious advantages, namely lower cost and easier acquisition. We adapted the inner and outer rings of the self-made device to a small incision in the abdominal wall, thus preventing leakage and ensuring a successful operation.

In patients with EP or UL, the operation time of SPL was longer than that of CL. Gasparri et al. also reported that the operation time of SPL was longer than that of $C L$ to treat EP [26], perhaps because the fixation of the self-made, multi-channel laparoscopy device is unreliable. Nonetheless, the procedure showed efficacy and safety after completion. The advantages of SPL in the present study included decreased blood loss, reduced pain, and fast recovery, which corresponds with published literature [27]. However, Schmitt et al. showed no significant difference between SPL and CL in terms of postoperative pain at 6 and $24 \mathrm{~h}$, blood loss, and mean length of hospital stay after adnexal surgery [28], perhaps because the SPL procedures were performed by skilled surgeons with vast experience in minimally invasive surgery in that study, whereas the senior surgeon and assistant surgeon in the present study were unfamiliar with the self-made SPL technique. In addition, the SPL incision site was concealed after surgery in the present study, perhaps because the procedure only requires a single incision in the umbilicus. Some authors have suggested that SPL is associated with a better cosmetic outcome than CL $[12,29,30]$. However, few studies have compared SPL with $C L$ in patients with EP or UL, and the present study offers preliminary evidence for the application of SPL in gynecological diseases.
Specimen retrieval remains an issue in minimally invasive surgical techniques, especially in benign gynecological surgery. In the present study, the specimens were removed directly through the umbilical incision using rotatory resection. An incision protective sleeve was used in the umbilical hole during the operation; it protected the wound and prevented iatrogenic pollution or infection. Moreover, the glove could be disconnected from the wound retractor to exteriorize the specimens; thus, the specimen could be easily extracted. The disadvantages of the selfmade SPL technique include potential glove bulging or piercing by a needle or instruments, especially during long operations. For this reason, a double layer of gloves may be used in future. Another disadvantage of self-made SPL is that the procedure results in a larger wound than with commercial port systems when extracting bulky specimens or multiple specimens. This may be because the self-made device lacks a fixed point or fulcrum. Numerous studies have suggested that using posterior colpotomy to extract surgical specimens during laparoscopy or to perform an endoscopic operation has no negative effects on female sexual function [31-33]. In addition, Uccella et al. showed that using the vagina for mass extraction allows the introduction of larger endobags without increasing the width of the incision on the abdomen during laparoscopic surgery [34]. Thus, the transvaginal approach to extracting multiple specimens during self-made SPL requires further research.

In addition, the introduction of ancillary trocars into the abdomen is a major challenge of laparoscopy, because it can cause injury to the inferior epigastric artery. To avoid this, many efforts have been made to identify the safest introduction technique for ancillary trocars. Vitale et al. suggested introducing a yellow island to prevent inferior epigastric artery damage; this allows easier and safer trocar insertion during laparoscopic surgery [35]. In addition, Tinelli et al. indicated that the yellow island and tip entry can be guided using a suction cannula; this can solve many problems and prevent damage to organs and viscera during laparoscopic surgery [36]. Therefore, future studies should address whether the yellow island can be applied to prevent inferior epigastric artery injury during self-made SPL, especially in obese and dark-skinned patients, in whom it is difficult to localize the epigastric artery using the transillumination technique. 
SPL has some technical difficulties. In particular, triangulation is not possible because only a single incision is used, so surgeons may have a reduced sense of location and distance during the operation [37]. A serious problem of intraoperative SPL is bleeding, which is the most common motive for conversion to $\mathrm{CL}$ or open laparotomy [38]. Shetty et al. reported that the bleeding control procedure was limited because there was insufficient time and space for needle handling [39]. The umbilicus is not an available entry point in all patients. In particular, excessive BMI can influence the thickness of the abdomen wall [40]. We recommended some solutions to this, such as complete incision on the muscle and fascia, obtaining a suction tube of the appropriate inner diameter using elastic materials, choosing a camera and ports with a small diameter and 0-30 angle, choosing integrated instruments, and using barbed line sutures to avoid tying. The experience of the surgeon is also essential, and an estimated triangular region can be constructed based on the distances of devices. The surgical assistant can co-operate to guarantee a visual field by swing ing and rotating the camera as carefully as possible.

SPL is an innovative and emerging technique, whose value will be exhibited in many fields and departments after further exploration. The present single-center study had some limitations: (1) the sample size of the patients with EP, as well as that of the patients with UL, was small, so further multicenter studies with a larger number of subjects should be conducted; (2) the long-term physical recoveries were recorded and analyzed by the investigator, rather than objectively evaluated using quantified indicators; (3) detailed guidelines on the device standard, inclusion criteria, and operative procedure should be highlighted in future multi-center trials.

\section{Conclusions}

The intraoperative and postoperative conditions of SPL were better than those of CL, and SPL showed clinical efficacy, with advantages of minimal invasion, rapid recovery, and cost-effectiveness in patients with EP or UL. Thus, the self-made SPL technique described here offers a low-cost alternative to commercially available single-port devices.

\section{Conflict of interest}

The authors declare no conflict of interest.

\section{References}

1. Gaskins AJ, Missmer SA, Rich-Edwards JW, et al. Demographic, lifestyle, and reproductive risk factors for ectopic pregnancy. Fertil Steril 2018; 110: 1328-37.

2. Finlinson AR, Bollig KJ, Schust DJ. Differentiating pregnancies near the uterotubal junction (angular, cornual, and interstitial): a review and recommendations. Fertil Res Pract 2020; 6: 8.

3. Refaat B, Dalton E, Ledger WL. Ectopic pregnancy secondary to in vitro fertilisation-embryo transfer: pathogenic mechanisms and management strategies. Reprod Biol Endocrinol 2015; 13: 30.

4. Marion LL, Meeks GR. Ectopic pregnancy: history, incidence, epidemiology, and risk factors. Clin Obstet Gynecol 2012; 55 : 376-86.

5. Birch Petersen K, Hoffmann E, Rifbjerg Larsen C, et al. Cesarean scar pregnancy: a systematic review of treatment studies. Fertil Steril 2016; 105: 958-67.

6. Stewart EA, Cookson CL, Gandolfo RA, et al. Epidemiology of uterine fibroids: a systematic review. BJOG 2017; 124: 1501-12.

7. Stewart EA. Comparing apples to apples for fibroids. N Engl J Med 2020; 383: 489-90.

8. Kacperczyk J, Bartnik P, Romejko-Wolniewicz E, et al. Postmyomectomic uterine rupture despite cesarean section. Anticancer Res 2016; 36: 1011-3.

9. Donnez J, Dolmans MM. Uterine fibroid management: from the present to the future. Hum Reprod Update 2016; 22: 665-86.

10. Jacoby VL, Jacoby A, Learman LA, et al. Use of medical, surgical and complementary treatments among women with fibroids. Eur J Obstet Gynecol Reprod Biol 2014; 182: 220-5.

11. Vilos GA, Allaire C, Laberge PY, et al. The management of uterine leiomyomas. J Obstet Gynaecol Can 2015; 37: 157-78.

12. Pontis A, Sedda F, Mereu L et al. Review and meta-analysis of prospective randomized controlled trials (RCTs) comparing laparo-endoscopic single site and multiport laparoscopy in gynecologic operative procedures. Arch Gynecol Obstet 2016; 294: 567-77.

13. Scheib SA, Fader AN. Gynecologic robotic laparoendoscopic single-site surgery: prospective analysis of feasibility, safety, and technique. Am J Obstet Gynecol 2015; 212: 179.e171-178.

14. Lee IO, Yoon JW, Chung D, et al. A comparison of clinical and surgical outcomes between laparo-endoscopic single-site surgery and traditional multiport laparoscopic surgery for adnexal tumors. Obstet Gynecol Sci 2014; 57: 386-92.

15. Lee JH, Lee JR, Jee BC, et al. Safety and feasibility of a single-port laparoscopic adnexal surgery during pregnancy. J Minim Invasive Gynecol 2013; 20: 864-70.

16. Yang YS, Oh KY, Hur MH, et al. Laparoendoscopic single-site surgery using conventional laparoscopic instruments and glove port technique in gynecology: a single surgeon's experience. J Minim Invasive Gynecol 2015; 22: 87-93.

17. Schwitzguebel AJ, Bogoev M, Nikolov V, et al. Tennis elbow, study protocol for a randomized clinical trial: needling with and without platelet-rich plasma after failure of up-to-date rehabilitation. J Orthop Surg Res 2020; 15: 462.

18. Scheib SA, Thomassee M, Kenner JL. Enhanced recovery after surgery in gynecology: a review of the literature. J Minim Invasive Gynecol 2019; 26: 327-43. 
19. Song J, Le T, Hopkins L, et al. A comparison of disease recurrence between robotic versus laparotomy approach in patients with intermediate-risk endometrial cancer. Int J Gynecol Cancer 2020; 30: 160-6.

20. Bogani G, Leone Roberti Maggiore U, Rossetti D, et al. Advances in laparoscopic surgery for cervical cancer. Crit Rev Oncol Hematol 2019; 143: 76-80.

21. Sugimoto $M$, Tanaka K, Matsuoka $Y$, et al. da Vinci robotic single-incision cholecystectomy and hepatectomy using single-channel GelPort access. J Hepatobiliary Pancreat Sci 2011; 18: 493-8.

22. Agrusa A, Romano G, Cucinella G, et al. Laparoscopic, three-port and SILS cholecystectomy: a retrospective study. G Chir 2013; 34: 249-53.

23. Xie XF, Zhu JF, Song CL, et al. Mechanical evaluation of three access devices for laparoendoscopic single-site surgery. J Surg Res 2013; 185: 638-44.

24. Abdel-Karim AM, Elmissery M, Elsalmy S, et al. Laparoendoscopic single-site surgery (LESS) for the treatment of different urologic pathologies in pediatrics: single-center single-surgeon experience. J Pediatr Urol 2015; 11: 33.e31-37.

25. Sharma A, Dahiya P, Khullar R, et al. Single-incision laparoscopic surgery (SILS) in biliary and pancreatic diseases. Indian J Surg 2012; 74: 13-21.

26. Gasparri ML, Mueller MD, Taghavi K, et al. Conventional versus single port laparoscopy for the surgical treatment of ectopic pregnancy: a meta-analysis. Gynecol Obstetric Investig 2018; 83: 329-37.

27. Karabicak I, Karabulut K. Single port laparoscopic liver surgery: a minireview. World J Gastrointest Endosc 2016; 8: 444-50.

28. Schmitt A, Crochet P, Knight S, et al. Single-port laparoscopy vs conventional laparoscopy in benign adnexal diseases: a sys tematic review and meta-analysis. J Minim Invasive Gyneco 2017; 24: 1083-95.

29. Fagotti A, Bottoni C, Vizzielli G, et al. Laparoendoscopic single-site surgery (LESS) for treatment of benign adnexal dis ease: single-center experience over 3-years. J Minim Invasive Gynecol 2012; 19: 695-700.

30. Cho YJ, Kim ML, Lee SY, et al. Laparoendoscopic single-site surgery (LESS) versus conventional laparoscopic surgery for adnexal preservation: a randomized controlled study. Int J Women's Health 2012; 4: 85-91.

31. Bucher P, Ostermann S, Pugin F, et al. Female population perception of conventional laparoscopy, transumbilical LESS, and transvaginal NOTES for cholecystectomy. Surg Endosc 2011; 25: 2308-15.

32. Ghezzi F, Cromi A, Uccella S, et al. Transumbilical versus transvaginal retrieval of surgical specimens at laparoscopy: a randomized trial. Am J Obstet Gynecol 2012; 207: 112.e111-116.

33. Soyman Z, Kelekci S, Aydogmus S, et al. Transabdominal versus transvaginal specimen extraction in mini-laparoscopic surgery. J Obstet Gynaecol Res 2019; 45: 2400-6.

34. Uccella S, Cromi A, Bogani G, et al. Transvaginal specimen extraction at laparoscopy without concomitant hysterectomy: our experience and systematic review of the literature. J Minim Invasive Gynecol 2013; 20: 583-90.
35. Vitale SG, Gasbarro N, Lagana AS, et al. Safe introduction of ancillary trocars in gynecological surgery: the "yellow island" anatomical landmark. Ann Ital Chir 2016; 87: 608-11.

36. Tinelli A, Gasbarro N, Lupo P, et al. Safe introduction of ancillary trocars. JSLS 2012; 16: 276-9.

37. Karabicak I, Karabulut K, Yuruker S, et al. Single-port laparoscopic liver resection: largest Turkish experience. Indian J Surg 2017; 79: 111-5.

38. Gkegkes ID, lavazzo C. Single incision laparoscopic hepatectomy: a systematic review. J Minim Access Surg 2014; 10: 107-12.

39. Shetty GS, You YK, Choi HJ, et al. Extending the limitations of liver surgery: outcomes of initial human experience in a high-volume center performing single-port laparoscopic liver resection for hepatocellular carcinoma. Surg Endosc 2012; 26: 1602-8.

40. Kang SH, Park YS, Ahn SH, et al. Laparoendoscopic single-site bariatric surgery: a review of single-port laparoscopic and endoscopic bariatric treatments. J Obes Metab Syndr 2018; 27: 25-34.

Received: 9.12.2020, accepted: 26.03.2021. 